\title{
Severe Diabetic Ketoacidosis in Two Children with New Onset type 1 Diabetes after SARS-CoV-2 Infection
}

\author{
Caroline Ponmani ${ }^{1 *}$, Tony Hulse ${ }^{2}$ and Sophia D Sakka ${ }^{2,3}$ \\ ${ }^{1}$ Department of Paediatric Emergency Medicine, Barking Havering and Redbridge University Hospitals NHS Trust, UK \\ ${ }^{2}$ Department of Endocrinology and Diabetes, Evelina London Children's Hospital, UK \\ ${ }^{3}$ GKT School of Medical Education, King's College London Faculty of Life Sciences and Medicine, UK
}

Submission: March 27, 2021; Published: April 07, 2021

*Corresponding author: Caroline Ponmani, Consultant in Paediatric Emergency Medicine, Department of Paediatric Emergency Medicine, Barking Havering and Redbridge University Hospital NSH trust, Rom Valley Way RM7 0AG, London, UK

Abstract

Background: An increase in the incidence and the severity of diabetic ketoacidosis (DKA) in children with new onset type1 diabetes has been reported in the COVID-19 pandemic. Studies have linked this to SARS-CoV-2 although causality could not be established.

Methods: We report two children with new onset type 1 diabetes and serum autoantibodies against islet cells and glutamic acid decarboxylase who developed severe DKA following infection with SARS-CoV-2. Both tested positive for COVID -19 PCR from nasopharyngeal swabs and developed symptoms of severe DKA, shock and cerebral oedema. Both children deteriorated rapidly illustrating the combined synergistic effects of the inflammatory pathways of COVID -19 and DKA.

Results: Both children needed admission to intensive care. The first child had raised inflammatory and cardiac markers and was suspected to have Paediatric multisystem inflammatory syndrome temporarily associated with COVID-19 (PIMS-TS). The period of type 1 diabetes symptoms was short in the second child, she also presented with clinical and biochemical features of pancreatitis which improved with supportive management. She also presented with biochemical thyrotoxicosis at the time of diagnosis.

Conclusion: Our report shows the association between infection with SARS-CoV-2 and rapid decompensation to DKA possibly accelerating the development of new-onset type 1 diabetes in genetically predisposed children. Additionally, the second child had pancreatitis illustrating that SARS-CoV-2 can affect both the endocrine and the exocrine function of the pancreas. Enhanced vigilance and earlier intervention is needed to prevent decompensation to DKA.

Keywords: Diabetic ketoacidosis; Type 1 diabetes; SARS CoV-2; Acute hyperglycaemia; Glucose

Abbreviations: DKA: Diabetic Ketoacidosis; ED: Emergency Department; GP: General Practitioner; GCS: Glasgow Coma Scale; IV: Intravenous; PICU: Paediatric Intensive Care Unit

\section{Introduction}

Increase in the incidence and the severity of DKA in children with new onset type 1 diabetes during the COVID-19 pandemic has been reported from several published studies although causality could not be established [1-3]. Studies also indicate a link between COVID-19 and acute hyperglycaemia in adults infected with SARS-CoV-2 [4,5]. In vitro studies have shown that the virus itself may trigger the development of ketoacidosis via direct damage to pancreatic beta cells [6,7]. Our report shows the association between infection with SARS-CoV-2 and rapid decompensation to DKA in two children with new onset type 1 diabetes.

\section{Case 1}

A 10-year-old Caucasian girl presented to the Emergency Department (ED) in May 2020 with a two day history of difficulty in breathing and a one day history of lethargy. She presented to the general practitioner (GP) initially, a diagnosis of asthma was made and she was prescribed Salbutamol inhaler. The next day she developed slurred speech, was unable to walk and was brought to ED by parents. Parents reported that she had a fourmonth history of gradual weight loss and six weeks of polyuria and polydipsia (Figure 1). She presented with fluid refractory 


\section{Current Research in Diabetes \& Obesity Journal}

shock and a fluctuating Glasgow Coma Scale (GCS) of 11-13. Her $\mathrm{mEq} / \mathrm{l}$ and glucose of $37 \mathrm{mmol} / \mathrm{l}$. initial blood gas showed a $\mathrm{pH} 6.91$, base deficit of $27, \mathrm{HCO}_{3}$ of 2.6

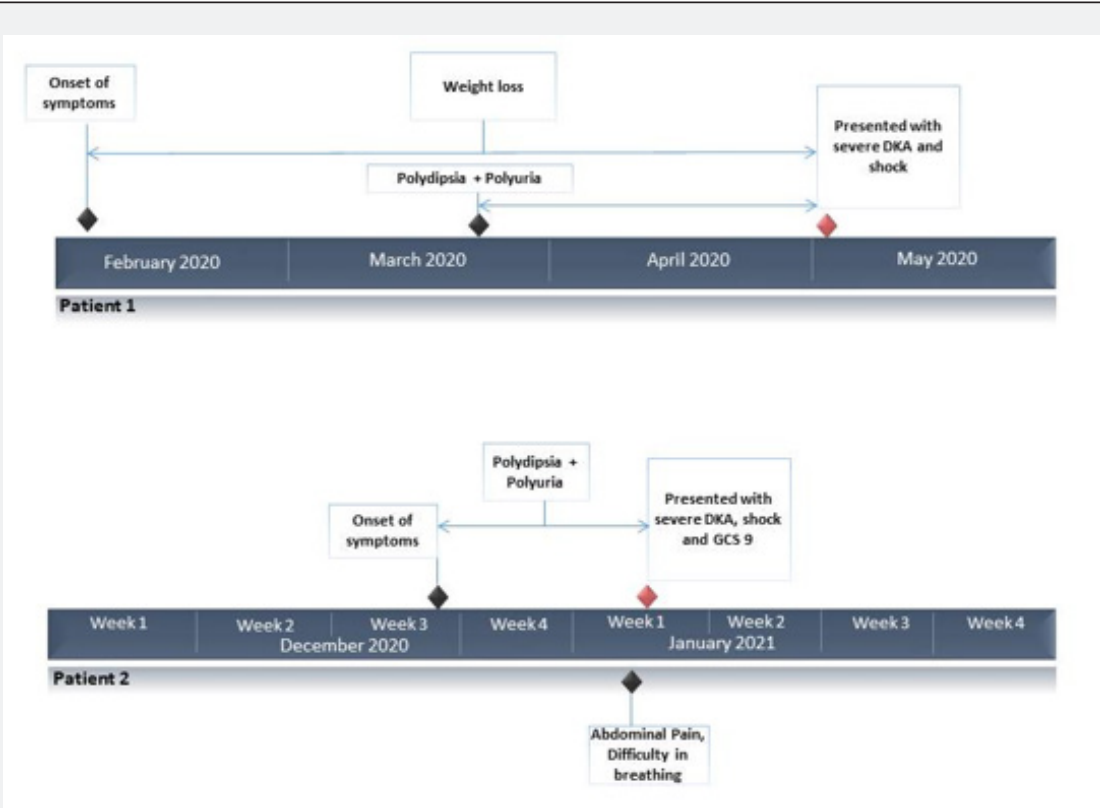

Figure 1: Timeline of patient one and two.

She was diagnosed with new onset type 1 diabetes with severe DKA and shock. She was suspected to have cerebral oedema. Intravenous (IV) fluids followed by insulin infusion were started, but went on to need inotropic support. She was also started on IV Ceftriaxone. PIMS-TS was suspected due to her clinical picture and raised inflammatory and cardiac markers [CRP: 215mg/L, WBC: 11.3 x 109 L, Procalcitonin: 5.32 ug/L (0-0.05), Ferritin: 490ug/L (14-101), troponin: 35 (0-13ng/L), D-dimers: 4.66 mg/L $(0-0.55)]$ and she was transferred to the Paediatric intensive care unit (PICU). She needed treatment in PICU for 4 days, her neurology improved with DKA management and she did not need hyperosmolar treatment. She did not receive treatment for PIMS -TS as she did not fulfil all the criteria.

CT head and echocardiography were normal. Her blood cultures were negative. Her nasopharyngeal swab was positive for COVID -19. On day 5, she was transferred to the paediatric ward, and subcutaneous insulin therapy was initiated. Over the course of the next few days her blood glucose levels stabilized. She received an educational programme on diabetes mellitus management and was discharged home on subcutaneous insulin injections. Her weight was $46 \mathrm{~kg}$, height was $153 \mathrm{~cm}$ and BMI was $19.52 \mathrm{~kg} / \mathrm{m}^{2}$. She had raised serum autoantibodies against islet cells and glutamic acid decarboxylase, confirming the diagnosis of type 1 diabetes. There is a strong family history of diabetes. Her father, paternal grandfather and both maternal grandparents have type 2 diabetes. Mother's first cousin has type 1 diabetes.

\section{Case 2}

A second child, a 13 year old Afro Caribbean girl presented to ED in January 2021 with a one day history of difficulty in breathing. She also went on to develop abdominal pain and vomiting and became increasingly lethargic over the day. She became unresponsive and was brought to ED. Parents reported that she had polyuria and polydipsia for 2 weeks (Figure 1). She was in shock and had a fluctuating GCS of 9-12. Her initial blood gas showed a pH 6.86, base deficit of 28.5, HCO3 of $6.4 \mathrm{mEq} / \mathrm{l}$ and glucose of $45 \mathrm{mmol} / \mathrm{l}$. Her WBC count was raised $37.6 \times 109 \mathrm{~L}$ with raised neutrophils of $31.1 \times 10^{9} \mathrm{~L}$, urea of $17.8 \mathrm{mmol} / \mathrm{L}$, Creatinine of $218 \mathrm{umol} / \mathrm{l}, \mathrm{HbA} 1 \mathrm{c}$ of $105 \mathrm{mmol} / \mathrm{mol}$,CRP of 7, amylase of 1200(30-100U/L), lipase of 314 IU/L(10-180 IU/L), C peptide of $435 \mathrm{pmpl} / \mathrm{L}$ and insulin of $14.6 \mathrm{miu} / \mathrm{L}$. She was diagnosed with new onset type 1 diabetes with severe DKA, shock and pancreatitis. IV fluids followed by insulin infusion were started. She was transferred to PICU. She improved with management of DKA, did not need hyperosmolar therapy and was transferred to the paediatric ward after 48 hours. Pancreatitis improved with supportive management, her repeat amylase was $392 \mathrm{IU} / \mathrm{L}$ and lipase was 328IU/L. Ultrasound abdomen was normal. Over the course of the next few days her blood glucose levels stabilized. She received education on diabetes mellitus management and was discharged home on subcutaneous insulin injections. Her blood culture did not show growth of any organisms. COVID -19 PCR was positive from nasopharyngeal swab. She had serum 
autoantibodies against islet cells and glutamic acid decarboxylase confirming the diagnosis of type 1 diabetes. At diagnosis she was also found to have raised fT4 $25.3 \mathrm{pmol} / \mathrm{L}(12.6-21 \mathrm{pmol} / \mathrm{L})$ with suppressed TSH of $0.01 \mathrm{mU} / \mathrm{L}(0.5-4.3 \mathrm{mU} / \mathrm{L})$, but with no symptoms of hyperthyroidism. They were repeated after DKA was treated and remained abnormal, along with positive TSH Receptor antibodies of $1.79 \mathrm{u} / \mathrm{ml}$ (normal range $<0.04 \mathrm{IU} / \mathrm{ml}$ ), confirming the diagnosis of Graves disease. Her weight was $44 \mathrm{~kg}$, height was $166 \mathrm{~cm}$ and BMI of $15.97 \mathrm{~kg} / \mathrm{m}^{2}$. There is a strong family history of diabetes, both maternal grandparents and paternal grandfather have type 2 diabetes.

\section{Discussion}

Analysis of the timeline of both cases raised the question - Is SARS-Co- 2 accountable for the incidence and the severity of DKA in the children?

Historically, viruses have been suspected to be the triggers in the onset and progress of type 1 diabetes in a genetically predisposed individual. However, it has been difficult to implicate them as critical infectious event that initiates autoimmunity predate clinical onset of type 1 diabetes by months to years [8]. Recent in vitro studies have shown that SARS-Co-2 itself may trigger the development of ketoacidosis via direct damage to pancreatic $\beta$-cell cells [6]

Investigation of the timeline of the onset and progress of symptoms in the first child showed that she had symptoms of type 1 diabetes (polyuria polydipsia and weight loss) preceding the onset of infection with SARS-CoV- 2. Infection with the virus appears to have precipitated DKA. In the second child the period of type 1 diabetes symptoms was of very short duration, she developed symptoms of difficulty in breathing, she also had severe epigastric pain and vomiting with a raised amylase and lipase and was diagnosed with pancreatitis illustrating the exocrine component of infection with SARS-CoV- 2 . Both children had type 1 diabetes antibodies and patient 2 has thyroid receptor antibodies, indicating the presence of an underlying autoimmunity making it less likely that the virus was causal for type 1 diabetes. However, the severity of DKA in both and the short duration of type 1 diabetes symptoms in the second child illustrates that infection with SARS-CoV-2 may have resulted in rapid loss of $\beta$-cells resulting in severe DKA. The inflammatory cascades of the COVID -19 infection and DKA may have acted synergistically contributing to the severity of the clinical manifestations in both children.

Delay in presentation to ED due to parental fears of COVID -19 resulting in DKA in children with new onset type 1 diabetes has been reported in some $[9,10]$ but not all studies [4]. There is an intrinsic delay in the recognition of paediatric diabetes due to the low volume presentation in children and nonspecific symptoms. Children have the ability to compensate making recognition difficult until they deteriorate rapidly. In the first patient, parents noticed polydipsia and increased sleeping but attributed these symptoms to boredom due to lockdown. Parents sought help appropriately when she developed symptoms of difficulty in breathing, initially from primary care where she was given salbutamol inhalers. She became increasingly lethargic over the next 24 hours and again parents correctly sought help bringing her to ED. The second child had a short period of symptoms of 2 weeks duration. Parents sought help from 111 when she developed difficulty in breathing then brought her to ED when she became unresponsive. We did not find delayed presentation due to parental fears of COVID -19 to be a significant factor in the decompensation to DKA in both children.

Diabetic patients have increased chances of developing autoimmune thyroid disease [11]. Thyrotoxicosis also occurs with increased frequency in diabetic children than in the general population, but it usually present after the diagnosis of diabetes. Autoimmune thyrotoxicosis at the time of type 1 diabetes presentation, like in case 2, is quite rare [12] and raised the question whether COVID-19 infection triggered an exacerbated autoimmune response.

\section{Conclusion}

We present for the first time a descriptive study of two children who presented with new onset diabetes, developed severe DKA after infection with SARS- CoV- 2 and tested positive for COVID-19 PCR on nasopharyngeal swabs. The observations from our study have important implications in emphasizing the need to raise public and clinician awareness for early recognition and prevention of DKA especially in informing clinicians who may see children in the early phase of new onset diabetes. Additionally the period of type 1 diabetes symptoms was very short in the second child, a variation which has been reported in in two other studies other study [2,3]. Further investigation is needed on whether children are presenting with a shorter duration of symptoms of type 1 diabetes in the pandemic. Whilst we illustrate that infection with SARS-CoV-2 contributed to DKA and accelerated the onset of diabetes, we acknowledge that more clinical research and additional qualitative and quantitative studies are needed. While we have progressed rapidly in advances in the treatment of paediatric type 1 diabetes the epidemiology is still shrouded in mystery, perhaps now is the time to learn from SARS $-\mathrm{CoV}-2$ and its proposed affinity for pancreatic $\beta$-cells. In the meantime public and clinician awareness campaigns emphasizing recognition of diabetic ketoacidosis remain key.

\section{Learning Points}

Severe DKA in children with new onset type 1 diabetes is reported for the first time with proven SARS-CoV-2 infection. Viral triggers in the onset and progress of new onset type 1 diabetes have always been suspected, now may be the time to learn from SARS - CoV- 2 and its proposed affinity for pancreatic $\beta$-cells. 


\section{Current Research in Diabetes \& Obesity Journal}

\section{Consent}

Both families have consented to the publication of the cases.

\section{Acknowledgement}

With grateful thanks to Yvette Redpath, Paediatric Emergency Department Clinical Fellow, Barking Havering and Redbridge NHS Trust, Romford, UK.

Saima Khan, Paediatric Senior House Officer, Barking Havering and Redbridge NHS Trust, Romford, UK.

Emma Jobson, Abby Billinghurst, Nicola Hammond, Staff Nurses Paediatric Emergency Department, Barking Havering and Redbridge NHS Trust, Romford, UK.

\section{References}

1. Rabbone I, Schiaffini R, Cherubini V, Maffeis C, Scaramuzza A (2020) Has COVID-19 Delayed the Diagnosis and Worsened the Presentation of Type 1 Diabetes in Children? Diabetes Care 43(11): 2870-2872.

2. Unsworth R, Wallace S, Oliver NS, Yeung S, Kshirsagar A, et al. (2020) New-Onset Type 1 Diabetes in Children During COVID-19: Multicenter Regional Findings in the U.K. Diabetes Care 43(11): e170-e171.

3. Ponmani C, Sakka SD, Wickramarachchi CS, Ajzensztejn M, Kanumakala S (2021) Characteristics of New-Onset Paediatric Type 1 Diabetes in the COVID-19 Pandemic - A Multicentre Perspective. Ann Pediatr 4(1): 1065.

4. Chen N, Zhou M, Dong X, Jieming Q Gong F, et al. (2020) Epidemiological and clinical characteristics of 99 cases of 2019 novel coronavirus pneumonia in Wuhan, China: a descriptive study. Lancet 395(10223): 507-513.
5. Wu J, Huang J, Zhu G, Wang Q Lv Q et al. (2020) Elevation of blood glucose level predicts worse outcomes in hospitalized patients with COVID-19: a retrospective cohort study. BMJ Open Diabetes Res Care 8(1): e001476.

6. Müller JA, Grob R, Conzelmann C Kruger J, Merle U, Steinhart J, et al. (2021) SARS-CoV-2 infects and replicates in cells of the human endocrine and exocrine pancreas. Nat Metab 3(2): 149-165.

7. Fignani D, Licata G, Brusco N, Nigi L, Grieco GE, et al. (2020) SARSCoV-2 Receptor Angiotensin I-Converting Enzyme Type 2 (ACE2) Is Expressed in Human Pancreatic $\beta$-Cells and in the Human Pancreas Microvasculature. Front Endocrinol (Lausanne) 11: 596898.

8. Filippi CM, Von Herrath MG (2008) Viral trigger for type 1 diabetes: pros and cons. Diabetes 57(11): 2863-2871.

9. Elbarbary NS, Dos Santos TJ, de Beaufort C, Agwu JC, Calliari LE, et al. (2020) COVID-19 outbreak and pediatric diabetes: Perceptions of health care professionals worldwide. Pediatr Diabetes 21(7): 10831092.

10. Lynn RM, Avis JL, Lenton S, Amin-Chowdhury Z, Landhani SN (2020) Delayed access to care and late presentations in children during the COVID-19 pandemic: a snapshot survey of 4075 paediatricians in the UK and Ireland. Arch Dis Child 106(2): e8.

11. Lorini R, d'Annunzio G, Vitali L, Scaramuzza A (1996) IDDM and autoimmune thyroid disease in the pediatric age group. J Pediatr Endocrinol Metab 9(Suppl 1): 89-94.

12. Devarajan R, Senuri K (2019) Concurrent presentation of thyroid storm and diabetic ketoacidosis: a systematic review of previously reported cases. BMC Endocr Disord 19(1): 49.

Your next submission with Juniper Publishers
will reach you the below assets
- Quality Editorial service
- Swift Peer Review
- Reprints availability
- E-prints Service
- Manuscript Podcast for convenient understanding
- Global attainment for your research
- Manuscript accessibility in different formats
( Pdf, E-pub, Full Text, Audio)
- Unceasing customer service
Track the below URL for one-step submission
https://juniperpublishers.com/online-submission.php

